\title{
Colloidal crystal growth at externally imposed nucleation clusters
}

\author{
Sven van Teeffelen ${ }^{\text {* }}$ Christos N. Likos, and Hartmut Löwen \\ Institut für Theoretische Physik II, Weiche Materie, \\ Heinrich-Heine-Universität Düsseldorf, D-40225 Düsseldorf, Germany
}

(Dated: November 10, 2018)

\begin{abstract}
We study the conditions under which and how an imposed cluster of fixed colloidal particles at prescribed positions triggers crystal nucleation from a metastable colloidal fluid. Dynamical density functional theory of freezing and Brownian dynamics simulations are applied to a twodimensional colloidal system with dipolar interactions. The externally imposed nucleation clusters involve colloidal particles either on a rhombic lattice or along two linear arrays separated by a gap. Crystal growth occurs after the peaks of the nucleation cluster have first relaxed to a cutout of the stable bulk crystal.
\end{abstract}

PACS numbers: 82.70.Dd, 81.10.Aj, 64.70.Dv, 81.10.-h

While important steps towards a quantitative understanding of homogeneous crystal nucleation out of the melt have been made in the past decade (for recent reviews, see [1, 2]), work on the molecular principles of heterogeneous nucleation is still at its infancy [3, 4, 5]. Colloidal suspensions have served as excellent model systems for nucleation, since the crystallization process is typically much slower than in their molecular counterparts and the critical nucleus can be detected in real space [6. By using external fields, e.g., optical tweezers, it is possible to fix a cluster of colloidal particles and watch directly its impact on the rest of the colloidal suspension. If the crystal phase is slightly off-coexistence and the fluid is stable, it is possible to generate crystalline layers around such a cluster [7, 8, 9].

In this Letter we study crystal growth processes into a metastable fluid. A cluster of fixed colloidal particles, which could act as a seed for heterogeneous crystal nucleation is arranged within the metastable melt. Whereas in homogeneous nucleation such clusters spontaneously form by means of thermal fluctuations, here they are externally imposed. We investigate whether they serve as initiators of crystal growth processes. Our motivation for this study is twofold: first, by imposing a seed cluster one can steer the crystallization behavior. Second, offering complex cluster structures could lead to unexpected dynamical scenarios of crystal growth.

We approach the problem using classical density functional theory (DFT) of freezing which is a microscopic approach to crystallization [10, 11. DFT can be extended to describe dynamics in strongly inhomogeneous Brownian fluids [12, 13, 14, 15]. Here it is put forward as the first full microscopic approach to the dynamics of crystallization. Our DFT results are backed by Brownian dynamics (BD) computer simulations. In principle, the dynamical DFT is superior to phase-field crystal theories of nucleation [16], which operate on more coarsegrained length and time-scales and need phenomenological mobilities as an input. Therefore our results provide benchmark data to test the validity of more approximate theories.

In detail, we study a model for a two-dimensional suspension of superparamagnetic colloids, exposed to an external magnetic field which tunes their parallel dipole moments [17, 18. By using additional fields, such as optical tweezers, certain particles can be fixed in the suspension [19, 20]. We first consider a stable fluid phase, realized for a weak magnetic field. In this fluid suspension, colloidal particles are placed by optical tweezers into prescribed positions forming a cluster. Then the magnetic field is suddenly increased rendering the fluid metastable with respect to the stable hexagonal crystal and the tweezers are released. Two different kinds of cluster geometries are considered: In the first setup we study hexagonal clusters that are cut out of a perfect rhombic lattice while in the second setup two sets of linear crystalline arrays, separated by a gap, are examined.

As a result, we observe that the kinetic pathway of the system is a two-stage dynamical process: first, on a sub-Brownian time scale, the peak positions of the externally imposed nucleation cluster relax towards a cutout of the stable bulk crystal. Then, on a Brownian time scale, there are two further possibilities: either the relaxed cluster acts as a nucleation seed for further complete crystal growth or it dies out completely without stimulating further crystallization. Whether crystal growth occurs or not depends delicately on the compatibility of the initial cluster geometry with that of the stable bulk crystal in terms of strain energy.

Our system is characterized by the pairwise interaction potential $u(r)=u_{0} / r^{3}$, where $u_{0}$ is the interaction strength. For the specific realization of twodimensional paramagnetic colloids of susceptibility $\chi$ exposed to a perpendicular magnetic field $\mathbf{B}$, we have $u_{0}=(\chi \mathbf{B})^{2} / 2$. The thermodynamics and structure depend only on one dimensionless coupling parameter $\Gamma=u_{0} \rho^{3 / 2} / k_{B} T$, where $\rho$ is the average one-particle density and $k_{B} T$ is the thermal energy.

It has been shown [12, 13, 14, that the static, classical DFT can be given an extension to dynamics to describe 
overdamped, time-dependent, out-of-equilibrium systems in terms of a deterministic, time-dependent, and ensemble averaged one-particle density $\rho(\mathbf{r}, t)$. The time evolution of $\rho(\mathbf{r}, t)$ is then governed by the continuity equation

$$
\frac{\partial \rho(\mathbf{r}, t)}{\partial t}=\frac{D}{k_{B} T} \nabla \cdot\left[\rho(\mathbf{r}, t) \nabla \frac{\delta F[\rho(\mathbf{r}, t)]}{\delta \rho(\mathbf{r}, t)}\right] .
$$

Here, $D / k_{B} T$ is the mobility coefficient originating from the solvent, ignoring hydrodynamic interactions.

The equilibrium phase diagram of the system under study has been obtained using classical DFT [21], which provides the intrinsic Helmholtz free energy functional $F[\rho(\mathbf{r})]$, a unique functional of the static one-particle density $\rho(\mathbf{r})$ of the system. The functional $F[\rho(\mathbf{r})]$ is minimized by the equilibrium one-particle density, where it takes the value of the system's intrinsic Helmholtz free energy. The density functional is typically split into the ideal gas, an excess, and an external part, $F[\rho(\mathbf{r})]=F_{\text {id }}[\rho(\mathbf{r})]+F_{\text {ex }}[\rho(\mathbf{r})]+F_{\text {ext }}[\rho(\mathbf{r})]$. The ideal part is $F_{\text {id }}[\rho(\mathbf{r})]=k_{B} T \int \mathrm{d} \mathbf{r} \rho(\mathbf{r})\left\{\ln \left[\rho(\mathbf{r}) \Lambda^{2}\right]-1\right\}$, with $\Lambda$ denoting the thermal de Broglie wavelength. $F_{\text {id }}$ is of completely entropic nature and leads to a simple diffusion term in Eq. (1). The excess part $F_{\text {ex }}$, originating from the correlations between the particles, is in this paper approximated by the ansatz of Ramakrishnan and Yussouff to the DFT [22]. It is expanded up to second order in terms of density difference $\Delta \rho=\rho(\mathbf{r})-\rho$ around a reference fluid, where the fluid density $\rho$ is chosen as the average density of the inhomogeneous system: $F_{\mathrm{ex}}[\rho(\mathbf{r})] \simeq$ $F_{\text {ex }}(\rho)-\frac{1}{2} k_{B} T \iint \mathrm{d} \mathbf{r} \mathrm{d} \mathbf{r}^{\prime} \Delta \rho(\mathbf{r}) \Delta \rho\left(\mathbf{r}^{\prime}\right) c_{0}^{(2)}\left(\mathbf{r}-\mathbf{r}^{\prime} ; \rho\right)$. Here $F_{\text {ex }}(\rho)$ and $c_{0}^{(2)}(\mathbf{r} ; \rho)$ are the excess free energy and the direct correlation function of the reference fluid of density $\rho$, respectively. Finally, the external part is simply given by $F_{\text {ext }}[\rho(\mathbf{r})]=\int \mathrm{d} \mathbf{r} \rho(\mathbf{r}) V(\mathbf{r})$, where $V(\mathbf{r})$ is the total external potential.

For both setups under study, the clusters of tagged particles are first, i.e., for times $t<0$, held fixed in a thermodynamically stable, equilibrated fluid of density $\rho$ at a coupling constant of $\Gamma_{<}=10$, which is well below the freezing transition at $\Gamma \simeq 35.7$ [23], obtained within the theory. For the equilibration of the fluid, Eq. (1) is numerically solved fixing the tagged particles by deep parabolic external potentials at the tagged particle positions - in an experiment this could be achieved by using optical tweezers 19 . At time $t=0$ we turn the external pinning potential off and, at the same time, instantaneously quench the system to a coupling constant $\Gamma_{>}=62.5$, which is well above the freezing transition and we observe the time evolution of the density field for times $\left(t / \tau_{B}\right) \lesssim 10$, where $\tau_{B}=(\rho D)^{-1}$ is the Brownian time scale. Eq. (1) is numerically solved applying a finite difference method. The dimensions $L_{x} \times L_{y}=n_{x} a \times n_{y}(\sqrt{3} / 2) a$ of the rectangular periodic box considered are chosen integer multiples $n_{x}, n_{y}$ of the lattice spacing $a=(2 / \sqrt{3})^{1 / 2} \rho^{-1 / 2}$ of the perfectly

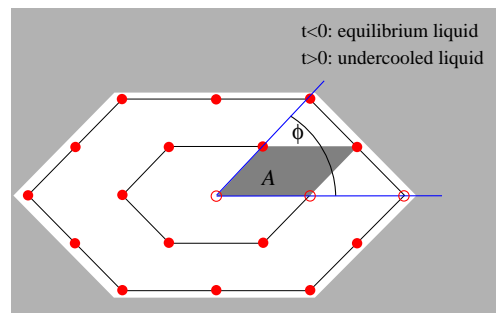

FIG. 1: Sketch of the imposed, rhombic nucleation cluster of 19 particles surrounded by a gray fluid. The angle $\phi$ between the spanning basis vectors and the area of a unit cell $A$ are also shown.

ordered hexagonal crystal.

The first setup under study comprises a rhombic nucleation seed of 19 tagged particles, arranged in a hexagon, as sketched in Fig. 1. The nucleus is characterized by the strain parameters $A$, the area of a unit cell which in the perfectly ordered hexagonal crystal equals $A=1 / \rho$, and $\phi$, the angle spanned by two of the nucleus axes. The size of the periodic rectangular box is $16 a \times 16(\sqrt{3} / 2) a$. In Fig. 2 snapshots of the time-evolving density field are shown exemplarily for two clusters cut out from two compressed hexagonal crystals with parameters $(A \rho=$ $0.7, \cos \phi=0.5)$ and $(A \rho=0.6, \cos \phi=0.5)$, respectively, at times $t / \tau_{B}=0,0.001,0.1,1.0$. While the former, less strongly compressed cluster grows into the equilibrium crystalline state, the latter collapses back into an undercooled, metastable fluid within $t / \tau_{B} \lesssim 0.1$. The growth dynamics of the stable nucleus is a two-stage process: In the first stage - on a sub-Brownian time scale $t \lesssim 0.002$ - the positions of the seed's density peaks move to a cutout of the thermodynamically stable bulk crystal. In the second stage - on the Brownian time scale — the system crystallizes out of the relaxed cluster.

Fig. 3 displays the "island" of growth in the $(A, \cos \phi)$ parameter space, i.e., the set of parameters, for which the nucleus grows for $t>0$. It is found that the "island" is nearly symmetric in $\cos \phi$, relative to the equilibrium value of $\cos \phi=0.5$ while it is asymmetric in unit cell area $A$ about the ideal value of $A=1 / \rho$. This asymmetry is qualitatively validated by $\mathrm{BD}$ simulations [25, 26.

Within the second setup we study the time evolution of a nucleation seed of two equal linear arrays along the $y$-direction, each comprising three infinite rows of hexagonally crystalline particles, which are separated by a gap, as can be seen from the density map for $t=0$ in Fig. 4 . These arrays, corresponding to an equilibrium crystal generated via a suitable external potential, are displaced relative to each other in $y$-direction by half a lattice spacing $\Delta y=a / 2$. In between the two crystalline arrays there is an empty stripe of width $\Delta x=\sqrt{3} a$ corresponding to one missing row of crystalline particles. In contrast to the first setup, the second setup corresponds to a configuration with a huge local, non-affine strain relative to a 


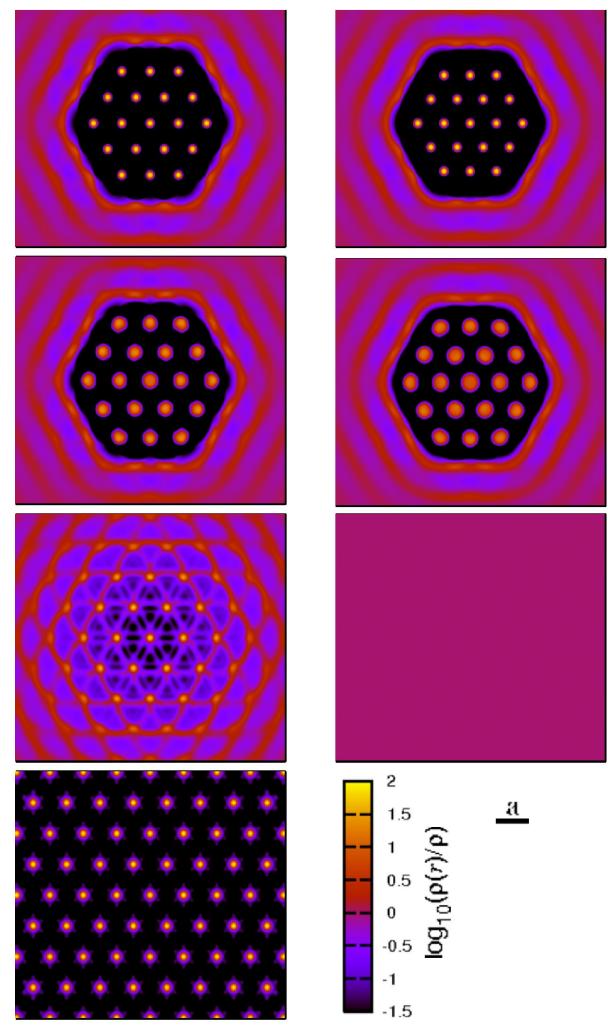

FIG. 2: Snapshots of the central region of the dimensionless density field $\rho(\mathbf{r}, t) / \rho$ of two colloidal clusters with strain parameters $A \rho=0.7, \cos \phi=0.5$ (left panel) and $A \rho=$ $0.6, \cos \phi=0.5$ (right panel) at times $t / \tau_{B}=0,0.001,0.1,1.0$ (from top to bottom; $t / \tau_{B}=1.0$ only for $A \rho=0.7$ ) [24]. Note that the images display only the system's central region of dimensions $L_{x} / 2 \times L_{y} / 2$.

perfect cut-out of a bulk crystal due to the gap.

In order to keep the gap free of particles during the equilibration of the surrounding fluid for times $t<0$ we employ an additional strong external potential in the region of the gap. The dimensions of the periodic box within which Eq. (1) is solved numerically are now given by $L_{x} \times L_{y}=64(\sqrt{3} / 2) a \times a$. Snapshots of the central region of the density field $\rho(\mathbf{r}, t)$ are shown in Fig. 4 for times $t / \tau_{B}=0,0.01,0.1,0.63,1.0$ after the quench.

Again, a two-stage dynamical scenario is observed: On a sub-Brownian time-scale of about $0.02 \tau_{B}$, the positions of the peaks drift to those of a perfect cut-out of the stable bulk crystal. This leads to a rapid filling of the gap. Then crystallization occurs on a Brownian time-scale. In Fig. 5 we plot the distances $x_{i}(t)$ of the three crystalline density peaks and the distance of the crystal front $x_{f}(t)$ with respect to the center of the gap as a function of time. The latter is taken as the inflection point of the envelope function of the $y$-averaged density field. The theoretical curves are compared to BD simulation data of the same setup [26] obtained by averaging over the

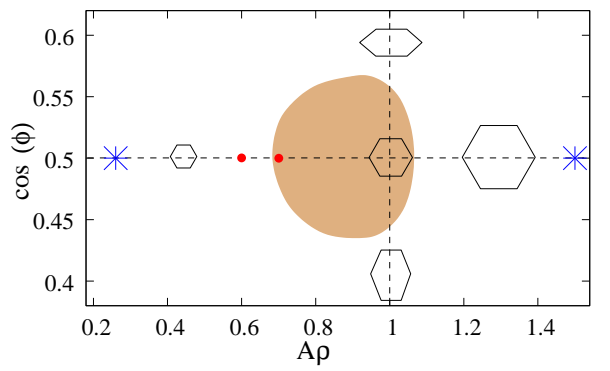

FIG. 3: Stability "island" of the imposed nucleation cluster of 19 particles according to Fig. 1. The shaded region separates the growth from the no-growth situation. The (blue) stars display the according boundaries for fixed $\cos \phi=0.5$ obtained from BD computer simulation. The hexagon symbols indicate the way the seeds are deformed in the different regions of the parameter space. The (red) dots indicate the configurations for the snapshots in Fig. 2.
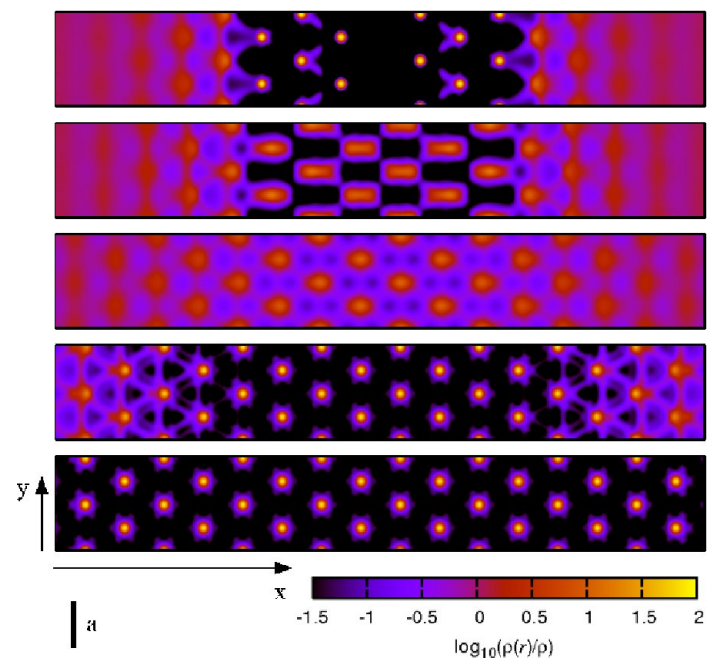

FIG. 4: Snapshots of the central region of the dimensionless density field $\rho(\mathbf{r}, t) / \rho$ of a linear nucleus of two times three infinite rows of hexagonally crystalline particles, separated by a gap, at times $t / \tau_{B}=0,0.01,0.1,0.63,1.0$ (from top to bottom) 24. Note that the images display twice the system's central region of dimensions $L_{x} / 4 \times 2 L_{y}$ for better visibility.

particle positions of 24.000 independent simulation runs. The two-stage picture is clearly confirmed.

In conclusion, we have investigated by dynamical density functional theory whether and how an externally imposed cluster of fixed particles acts as a nucleation seed for crystal growth if the particles are released and the system is quenched instantaneously from a stable to a metastable bulk fluid. If the imposed cluster is not too much strained relative to a cut-out of the stable bulk crystal, it induces global crystallization. The kinetic pathway of the imposed cluster exhibits a twostage scenario: the cluster structure first relaxes towards an appropriate cut-out of the bulk crystal before further growth. This two-stage process is unexpected since it is 


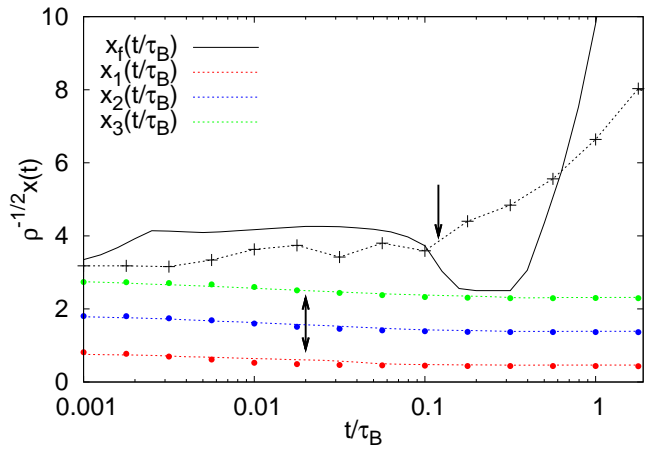

FIG. 5: Time evolution of the distance of the linear array's three density peaks $x_{i}(t)$ and of the crystal front $x_{f}(t)$ with respect to the center of the gap as a function of time. Dynamical density functional theory results (lines) are compared against Brownian dynamics simulation data (symbols; the dashed line connecting the crosses is a guide to the eye). The arrows indicate the typical time scales on which the relaxation of the $x_{i}$ is occurring and on which the crystal growth sets in, respectively.

reversed in larger clusters which contain quite a large portion of the stable bulk crystal. In the latter case crystal growth starts at the edges but the inner elastic distortion anneals on a much larger time scale. For higher undercoolings, i.e., larger $\Gamma_{>}$, the size of the stability island (Fig. 3) increases.

Our predictions can be verified by real-space experiments on two-dimensional superparamagnetic colloidal particles confined to the air-water interface in an external magnetic field [17, 18]. Qualitatively similar scenarios are expected for different repulsive interactions and in three spatial dimensions, which are relevant for nucleation and growth experiments in sterically and charge stabilized suspensions [8, 27, 28, 29]. In three dimensions, one may even induce the growth of metastable crystals and quasicrystals imposed by suitable nucleation seeds [30].

We thank N. Hoffmann, R. Blaak, M. Rex, and A. van Blaaderen for helpful discussions. This work has been supported by the DFG through the SFB TR6 (project section C3) and the DFT priority program SPP 1296.

* teeffelen@thphy.uni-duesseldorf.de

[1] S. Auer and D. Frenkel, Adv. Polym. Sci. 173, 149 (2005).

[2] E. Ruckenstein and Y. S. Djikaev, Adv. Colloid Interface Sci. 118, 51 (2005).

[3] R. P. Sear, J. Phys.: Condens. Matter 19, 033101 (2007).

[4] L. Gránásy, T. Pusztai, and J. A. Warren, J. Phys.: Condens. Matter 16, R1205 (2004).

[5] L. Gránásy, T. Pusztai, T. Borzsonyi, G. Toth, G. Tegze, J. A. Warren, and J. F. Douglas, J. Mater. Res. 21, 309 (2006).
[6] U. Gasser, E. R. Weeks, A. Schofield, P. N. Pusey, and D. A. Weitz, Science 292, 258 (2001).

[7] M. Heni and H. Löwen, Phys. Rev. Lett. 85, 3668 (2000).

[8] D. L. J. Vossen, A. van der Horst, M. Dogterom, and A. van Blaaderen, Rev. Sci. Instr. 75, 2960 (2004).

[9] A. Cacciuto and D. Frenkel, Phys. Rev. E 72, 41604 (2005).

[10] Y. Singh, Phys. Rep. 207, 351 (1991).

[11] J. Wu, AIChE J. 52, 1169 (2006).

[12] U. M. B. Marconi and P. Tarazona, J. Chem. Phys. 110, 8032 (1999).

[13] J. Dzubiella and C. N. Likos, J. Phys.: Condens. Matter 15, L147 (2003).

[14] A. J. Archer and R. Evans, J. Chem. Phys. 121, 4246 (2004).

[15] A stability analysis of undercooled fluids in three spatial dimensions with respect to periodic density waves was performed by B. Bagchi, Physica A 145, 273 (1987).

[16] K. R. Elder, M. Katakowski, M. Haataja, and M. Grant, Phys. Rev. Lett. 88, 245701 (2002).

[17] K. Zahn, J. M. Méndez-Alcaraz, and G. Maret, Phys. Rev. Lett. 79, 175 (1997).

[18] R. Haghgooie and P. S. Doyle, Phys. Rev. E 72, 011405 (2005).

[19] M. Köppl, P. Henseler, A. Erbe, P. Nielaba, and P. Leiderer, Phys. Rev. Lett. 97, 208302 (2006).

[20] A. Pertsinidis and X. S. Ling, Phys. Rev. Lett. 87, 98303 (2001); Nature 413, 147 (2001).

[21] S. van Teeffelen, C. N. Likos, N. Hoffmann, and H. Löwen, Europhys. Lett. 75, 583 (2006).

[22] T. V. Ramakrishnan and M. Yussouff, Phys. Rev. B 19, 2775 (1979).

[23] H. Löwen, C. N. Likos, L. Assoud, R. Blaak, and S. van Teeffelen, Philos. Mag. Lett. 87, 847 (2007).

[24] See http://www2.thphy.uni-duesseldorf.de/ teeffelen for movies of the time evolution of $\rho(\mathbf{r}, t)$ for both setups under study.

[25] In the Brownian dynamics simulations, the following cluster criterion for crystal growth was chosen. A particle is defined to be crystalline if it has six nearest neighbors according to the Voronoi construction. Neighboring particles $i$ and $j$ are assigned to the same crystalline cluster if $\left|\operatorname{Re}\left[\Psi_{6}^{i} \Psi_{6}^{j *}\right]\right| /\left|\Psi_{6}^{i} \Psi_{6}^{j *}\right| \lesssim 0.32$, where $\Psi_{6}^{i}$ is particle $i$ 's complex bond order parameter. Crystal growth is defined if the average number of crystalline particles belonging to the cluster which contains the seed's innermost particle at $t / \tau_{B}=0.1, N_{c}$, exceeds $N_{\min }=23$. For the strain parameters $\cos \phi=0.5, \rho A=0.2,0.4,0.6,0.8,1,1.2,1.4,1.6$ we performed 10.000 independent runs each and measured $N_{c}$ as a function of $A$. By linear interpolation the boundaries of the stability island for $\cos \phi=0.5$ were determined $A_{\min } \simeq 0.26, A_{\max } \simeq 1.5$.

[26] Since freezing in the simulation sets in at $\Gamma \simeq 12$ [18] we quench the simulated system from $\Gamma_{<}=5$ to $\Gamma_{>}=20$.

[27] H. J. Schöpe, G. Bryant, and W. van Megen, Phys. Rev. Lett. 96, 175701 (2006).

[28] P. Wette, H. J. Schöpe, and T. Palberg, J. Chem. Phys. 123, 174902 (2005).

[29] V. W. A. de Villeneuve, R. P. A. Dullens, D. G. A. L. Aarts, E. Groeneveld, J. H. Scherff, W. K. Kegel, and H. N. W. Lekkerkerker, Science 309, 1231 (2005).

[30] D. Zander, R. Janlewing, A. Rüdiger, and U. Köster, Mater. Sci. Forum 307, 25 (1999). 